\title{
Physical Activity, Ambulation, and Motor Impairment Late after Stroke
}

\author{
Anna Danielsson, ${ }^{1,2}$ Carin Willén, ${ }^{1}$ and Katharina Stibrant Sunnerhagenn ${ }^{1,3}$ \\ ${ }^{1}$ Institute of Neuroscience and Physiology/Rehabilitation Medicine, Sahlgrenska Academy, \\ University of Gothenburg, 41345 Göteborg, Sweden \\ ${ }^{2}$ Physiotherapy and Occupational Therapy, Sahlgrenska University Hospital, 41345 Göteborg, Sweden \\ ${ }^{3}$ Sunnaas Rehabilitation Hospital/Nesodden and Faculty of Medicine, University of Oslo, Norway \\ Correspondence should be addressed to Anna Danielsson, anna.danielsson@neuro.gu.se
}

Received 19 May 2011; Revised 15 July 2011; Accepted 19 July 2011

Academic Editor: Gert Kwakkel

Copyright (C) 2012 Anna Danielsson et al. This is an open access article distributed under the Creative Commons Attribution License, which permits unrestricted use, distribution, and reproduction in any medium, provided the original work is properly cited.

\begin{abstract}
Objective. To assess walking capacity and physical activity using clinical measures and to explore their relationships with motor impairment late after stroke. Subjects. A nonrandomised sample of 22 men and 9 women with a mean age of 60 years, 7-10 years after stroke. Methods. Fugl-Meyer Assessment, maximum walking speed, 6 min walk test, perceived exertion, and heart rate were measured, and the Physiological Cost Index was calculated. Physical activity was reported using The Physical Activity Scale for the Elderly. Results. Mean (SD) 6 min walking distance was $352( \pm 136) \mathrm{m}$, and Physiological Cost Index was $0.60( \pm 0.41)$. Selfreported physical activity was $70 \%$ of the reference. Motor impairment correlated with walking capacity but not with the physical activity level. Conclusion. It may be essential to enhance physical activity even late after stroke since in fairly young subjects both walking capacity and the physical activity level were lower than the reference.
\end{abstract}

\section{Introduction}

Ambulation is an essential part of daily physical activity. After stroke, about $65 \%$ of survivors have reduced ambulatory capacity [1] and after 6 months 50\% still have impaired muscle function [2]. Damage of motor and sensory pathways results in altered motor function [3] and, over time, intramuscular changes $[4,5]$. A reduction in active muscle mass may partly explain why peak oxygen uptake $\left(\mathrm{VO}_{2}\right)$ can be reduced to half of that of age-matched controls [4].

Impaired muscle function implies increased mechanical work and reduced walking speed to less than half of reference values [6]. A low walking speed together with poor muscle function and low aerobic capacity may result in a twofold increase in energy costs, defined as $\mathrm{VO}_{2}$ per unit distance walked $[6,7]$. Several studies confirmed a relationship between walking speed and motor impairment and some showed relationships between aerobic capacity and activity assessments [8].

A high energy cost of walking might affect the ability to perform daily activities and participation and thereby lead to a vicious circle where physical activity is avoided. In one study, stroke subjects walked $50 \%$ of the daily amount of matched sedentary persons' step counts and used $75 \%$ of their $\mathrm{VO}_{2}$ peak for walking at a submaximal rate [9].

Physical activity and exercise are significant in disease prevention, and a low daily physical activity level may involve a general health risk $[4,10]$. A survey in the USA revealed that $56 \%$ of people with disabilities were not engaged in physical activity and a study using focus groups found multifactorial reasons for this [11]. Studies on the actual physical activity level after stroke are few and are mostly performed in elderly populations [12]. A recent stroke study found very low daily activity levels as measured by accelerometer counts and selfreports, which could explain the poor quality of life to a certain extent [13].

One hypothesis is that the level of physical activity late after stroke is lower than in a nonstroke population and that walking capacity, general physical activity, and motor impairments are associated. The aim of the current study was to use clinically applicable methods to assess walking habits and capacity, energy costs, and physical activity levels in 
a fairly young sample several years after stroke and to explore whether or not motor impairment was associated with walking capacity and self-reported physical activity levels.

\section{Materials and Methods}

Fifty-four persons with a first event of stroke who had taken part in a previous study at a rehabilitation unit [14] were invited by post, followed by a telephone call, to participate. The criteria for participation were stroke according to the WHO definition, $>18$ years of age, the ability to communicate in Swedish, and to walk with or without a walking aid without personal assistance for 6 minutes. The study was approved by the Regional Ethical Review Board/Gothenburg and the participants gave their informed, written consent.

The subjects were asked to refrain from nicotine and coffee for at least 2 hours before the test. Two tests of walking capacity were performed. First, the maximum walking speed was measured on a $30 \mathrm{~m}$ track in an undisturbed corridor. The test of maximum walking speed has been shown to be reliable after stroke [8], and the $30 \mathrm{~m}$ distance was chosen in order to make comparisons with age- and sex-matched reference values for this distance, taken from a populationbased sample of people living in the same recruitment area as the stroke subjects [15]. A heart rate (HR) monitor (Polar S625X, Polar Elektro Oy, Kempele, Finland) with a storage function was attached with a chest strap, and the resting HR was measured while each subject sat in silence for 10 minutes; the mean of the last 5 minutes was used as the baseline value. As a second test of capacity, each subject was then instructed to walk at self-selected speed round a cone at each end of the $30 \mathrm{~m}$ walking track and to cover as much distance as possible in 6 minutes (6MWT) [16] while the HR was recorded. Each subject was asked to rate the perceived exertion on the Borg CR10 [17] scale after stopping, and the distance covered was estimated to the nearest metre. Two 6MWT tests were carried out separated by seated rest for 10 minutes or until return to the baseline HR. The longest distance of the two 6MWT was chosen for analysis, and each individual's $6 \mathrm{MWT}$ distance was compared to a reference value estimated by a gender-specific equation correcting for age, height, and weight [18]. The energy cost was estimated using the Physiological Cost Index (PCI) based on the relationship between oxygen consumption and heart rate [19], which has been investigated regarding reliability after stroke $[20,21]$ and considered to give a rough measure of the energy cost. Dividing the difference between walking and resting HR by the walking speed in $\mathrm{m} / \mathrm{min}$ gives the PCI value expressed as heartbeats $/ \mathrm{m}$. To ensure a steady state, the mean of the last 3 minutes of walking was used as the walking HR value.

Weight and height were recorded and the body mass index (BMI) was calculated. Medical problems besides stroke were reported using the Self-Administered Comorbidity Questionnaire [22] and any current medication that could possibly affect the heart rate was recorded.

The motor impairment of the affected leg was assessed using the Fugl-Meyer Sensorimotor Assessment [23], where the maximum score of 34 indicates good performance.
Each participant was interviewed using the following questionnaires: the frequency of continuous outdoor walking distances during the last 3 months was covered by the Walking Habit Score comprising five questions from a questionnaire on walking ability [24]. The answers were divided into two groups: one that was classified as inactive walkers who never or at most once a week walked a distance of $500 \mathrm{~m}$, and another group that walked $500 \mathrm{~m}$ or further more often. Leisure, household, and work-related physical activities during the most recent week were assessed using the Physical Activity Scale for the Elderly (PASE) [25]. The PASE was originally developed through a large population study on middle-aged and elderly people where self-reported physical activity was validated against accelerometer counts and each activity was weighted according to metabolic equivalents. By multiplying each activity's weight by time, a total score is calculated; a maximum score is not specified. In the original study [25], the test-retest reliability was $r 0.68-0.84$ and the PASE was found to be valid in elderly people with disabilities [26] and has been used for stroke subjects [27]. In the present study, each individual's PASE value was compared to ageand sex-matched normative values taken from a populationbased sample of 113 persons in age cohorts between 40 and 69 years of age, living in the same recruitment area as the study subjects.

2.1. Statistics. Descriptive statistics were given as the mean and standard deviation (SD) for continuous data and the median and interquartile range (IQR) for ordinal data. Differences between participants' values and reference values were analysed using a paired $t$-test, and any subgroup differences were explored using the Mann-Whitney $U$-test. Spearman's rho was calculated to investigate correlations between variables. The significance level was set at $P<0.05$.

\section{Results}

Thirty-one persons between 36 and 73 years of age volunteered for this study a median of 8 years (range 7-10) after the first stroke event, and only one had suffered a second stroke. Sixteen persons refrained from participating, five declined due to transportation problems, one did not understand Swedish, and one could not be reached. The people who were included and excluded did not differ in sex, age, or length of hospital stay in the early stage. Demographic and clinical data of the participants are given in Table 1 .

The Fugl-Meyer motor score in the affected leg ranged from 14 to 34 (Table 1). The maximum walking speed measured over $30 \mathrm{~m}$ ranged from 0.3 to $2.3 \mathrm{~m} / \mathrm{s}$, which corresponded to $62 \%$ of the sex- and age-matched reference values [15], and the 6MWT distance varied between 93 and $577 \mathrm{~m}$, corresponding to $52 \%$ of the calculated reference values [18] (Table 2). At the end of the 6MWT, the median (IQR) perceived exertion was $2(2-4)$ and the mean (SD) heart rate was $96( \pm 18)$ beats/min. Eight participants used a cane, two used a walker, and seven used an ankle-foot orthosis during the walking tests.

The energy cost estimated using the PCI showed no statistically significant differencebetween the two 
TABle 1: Demographic and clinical characteristics $(n=31)$.

Sex, female/male, $(n)$

$9 / 22$

Age, (y), mean (SD)

$59.7( \pm 8.1)$

Body mass index, mean (SD)

$27( \pm 4.7)$

Cerebral infarction/cerebral

haemorrhage/cerebellar haemorrhage $(n)$

$18 / 10 / 3$

Right/left/bilateral lesion $(n)$

$15 / 15 / 1$

Time since stroke (y)

Cardiovascular disease/pulmonary

disease/diabetes/musculoskeletal

$20 / 3 / 7 / 16 / 11$

pain/depression $(n)$

Smoking $(n)$

6

Beta blocker/calcium channel blocker/ACE

inhibitor/angiotensin antagonist/alpha

$15 / 9 / 6 / 4 / 2 / 5$

blocker/diuretics $(n)$

FMA, median (IQR)

$29(22-34)$

Walking aid, $(n)$

$14^{\mathrm{a}}$

Ankle-foot orthosis $(n)$

7

Wheelchair $(n)$

$4^{\mathrm{b}}$

SD: standard deviation: FMA: Fugl-Meyer Assessment. Motor score lower extremity, maximum 34 . IQR: interquartile range: ${ }^{\mathrm{a}}$ occasional use in four subjects: ${ }^{\mathrm{b}}$ occasional use.

TABle 2: Six minutes walking distance, maximum walking speed, energy cost, physical activity, and their associations with motor impairment of the affected lower extremity $(n=31)$.

\begin{tabular}{lcc}
\hline & & Spearman's rho \\
\hline 6MWT distance $(\mathrm{m})$, mean $(\mathrm{SD})$ & $352( \pm 136)$ & $0.80^{* *}$ \\
$\begin{array}{l}\text { Maximum speed over } 30 \mathrm{~m} \\
(\mathrm{~m} / \mathrm{s}), \text { mean }(\mathrm{SD})\end{array}$ & $1.30( \pm 0.57)$ & $0.82^{* *}$ \\
PCI (beats/m), mean (SD) & $0.60( \pm 0.41)$ & $-0.57^{* *}$ \\
PASE, mean (SD) & $124( \pm 67)$ & 0.24 \\
\hline
\end{tabular}

6MWT: 6-minute walk test: PCI: Physiological Cost Index: PASE: Physical Activity Scale for the Elderly. ${ }^{* *}$ correlation significant at $P<0.01$.

measurements $(P=0.678)$ and the PCI from the longest 6MWT distance chosen for analysis varied from 0.19 to 2.5 beats $/ \mathrm{m}$ (Table 2 ). Neither cardiopulmonary problems nor the use of beta blockers caused any statistically significant differences in the mean PCI values.

The physical activity level assessed using the PASE ranged from 31 to 241 (Table 2). In the 28 cases where age- and sex-matched reference values were available, the mean (SD) PASE score of $120( \pm 64)$ corresponded to $72 \%( \pm 66)$ of the reference sample. Seventeen participants never, or once a week at most, walked a continuous distance of $500 \mathrm{~m}$, as shown in Figure 1.

Motor impairment was significantly correlated (Table 2) with the maximum walking speed, the 6MWT distance, and the PCI, but not with the PASE. There was no significant correlation between the PASE and PCI.

Medical problems other than stroke-related problems were reported by 30 participants (Table 1 ), of whom 18 experienced activity limitations due to these. Of the 20

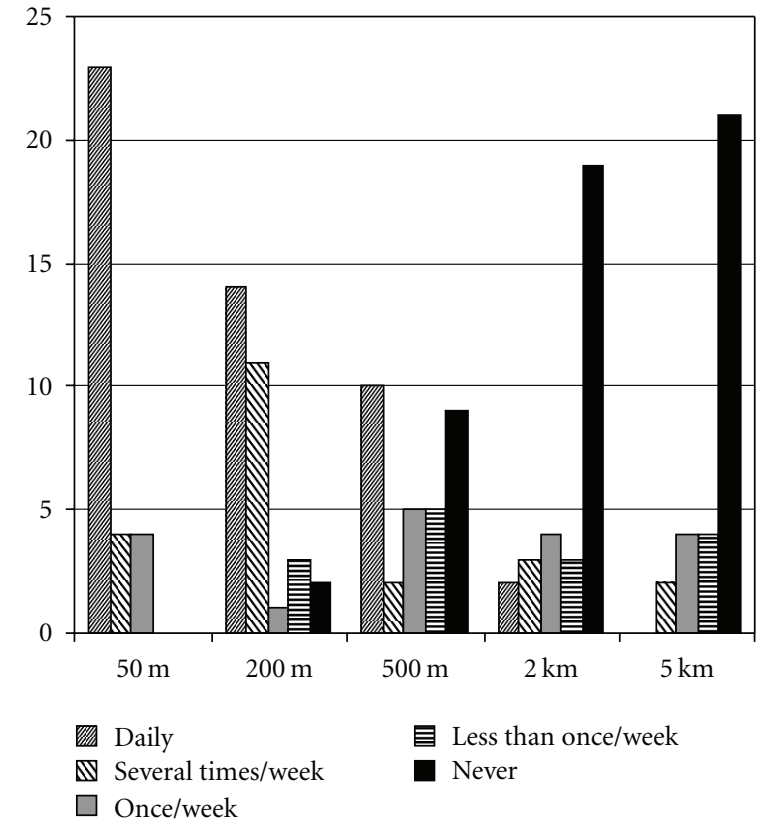

FIGURE 1: Frequency of walking specified continuous outdoor distances during the last 3 months.

participants with cardiopulmonary comorbidity, 5 stated activity limitations due to this. One subject had known atrial fibrillation with a frequency within normal boundaries.

\section{Discussion}

Our study of fairly young persons for whom several years had passed since stroke showed a reduced physical activity level and walking ability compared to healthy reference people. There was a large variation in motor impairment and walking capacity in the study group. The group's mean Body Mass Index indicated a tendency towards being overweight. The sample of the present study was small and highly selected, which limited the statistical power and generalisability of the results; however, our data adds information obtained using clinically feasible methods to previous findings.

Physical capacity, measured as the maximum walking speed, was well below normal as the mean was about $60 \%$ of the reference value. The mean 6MWT distance was half of the estimated normative values $[18,28]$, which was similar to the results found in another study at a later stage after stroke [29]. The energy cost measured using the PCI was approximately double compared to healthy people $[19,20$, 30 ] and comparable to another study of the energy costs involved in over-ground walking in stroke subjects [21]. However, both the heart rate and the ratings of perceived exertion indicated that the walking tests in the current study were not very strenuous, probably because of the low velocity. As the energy cost is expressed per unit of distance, a high value can be partly explained by a low walking speed, in addition to inefficient gait mechanics due to impaired muscle functioning. The PCI as a measure of energy cost is a clinical measure that is not as robust as measurements of $\mathrm{VO}_{2}$, which would have been preferred 
if portable equipment for gas analysis had been available. The measurement of heart rate may be unreliable in cases of arrhythmia, and several factors, for example, stress or other environmental factors, may influence the heart rate, which is the basis of the PCI equation. However, in the current study the presence of cardiopulmonary problems or beta blockade did not show any difference in the PCI. The fact that there was no difference between the groups with and without cardiopulmonary comorbidity may be explained by the relatively low walking speed that did not demand a high increase in heart rate compared to the resting HR. Findings published on the reliability and validity of PCI in general, as well as after stroke, vary [20, 21]; however, in the present study the values from the two tests were similar and currently PCI is one of the few methods applicable in a clinical setting, providing a rough measure of energy cost.

The level of physical activity measured by the PASE reached approximately $2 / 3$ of the reference values, which was somewhat higher than expected. A selection bias could be one possible explanation as more physically active participants could have volunteered and less-active people could have declined participation in the study. The walking activity reported, however, was approximately half of the frequency of the distances specified by healthy people in another study [24]. Based on the available data, it is difficult to say whether or not the participants fulfilled the health recommendations for a daily dose of physical activity as the intensity required is difficult to rate from the available data.

Both physical activity and walking habits were assessed by questionnaires; these might not be as accurate as accelerometer recordings, but in the current study no such equipment was accessible. Sufficient reliability of the self-report method was shown after stroke [31], although the method may be questionable due to memory, insight, and communication problems. However, we noticed that those who reported low walking distances on the questionnaires also gave a poorer performance in the $6 \mathrm{MWT}$, which strengthens the validity.

Our hypothesis that the level of motor impairment of the lower extremities is associated with walking capacity was supported by the high correlations between the Fugl-Meyer score and the speed and distance measures. However, the correlation between motor impairment and energy cost was lower. The energy cost was not associated with the general physical activity level, which could have been expected. An uncertain accuracy of the PCI measure may be one reason for this result. Another explanation could be that physical activity is a complex construct with many possible influencing factors. Coexisting disease may have an impact [32], and almost all of the participants in the present study had some comorbidity with $2 / 3$ feeling that this limited their activity levels. Depression was reported by $1 / 3$, but other factors that could determine performance such as fatigue, cognitive impairment [33], a previous experience of or attitudes towards physical activity were not investigated. Beyond physical capacity, walking habits might be influenced by the physical environment or by social or psychological factors [34].

\section{Conclusions}

In this sample of fairly young persons several years after stroke, walking capacity was approximately half the normal level, the energy cost of walking was twofold, and selfreported physical activity levels were lower compared to healthy reference people. Motor impairment was associated with walking capacity but not with physical activity level. There are indications that physical activity after stroke is undertaken less often than recommended, and for health reasons it may be important to assess and support feasible, regular physical activity sessions, even a long time after the end of rehabilitation.

\section{Acknowledgments}

This study was supported by the Norrbacka-Eugenia Foundation, the Swedish Stroke Association, the Council of Research and Development of Gothenburg and Southern Bohuslän, Hjalmar Svensson's Research Foundation, and John and Brit Wennerström's Foundation for Neurological Research.

\section{References}

[1] H. S. Jorgensen, H. Nakayama, H. O. Raaschou, and T. S. Olsen, "Recovery of walking function in stroke patients: the Copenhagen stroke study," Archives of Physical Medicine and Rehabilitation, vol. 76, no. 1, pp. 27-32, 1995.

[2] M. Kelly-Hayes, A. Beiser, C. S. Kase, A. Scaramucci, R. B. D'Agostino, and P. A. Wolf, "The influence of gender and age on disability following ischemic stroke: the Framingham study," Journal of Stroke and Cerebrovascular Diseases, vol. 12, no. 3, pp. 119-126, 2003.

[3] K. S. Sunnerhagen, U. Svantesson, L. Lönn, M. Krotkiewski, and G. Grimby, "Upper motor neuron lesions: their effect on muscle performance and appearance in stroke patients with minor motor impairment," Archives of Physical Medicine and Rehabilitation, vol. 80, no. 2, pp. 155-161, 1999.

[4] F. M. Ivey, R. F. Macko, A. S. Ryan, and C. E. Hafer-Macko, "Cardiovascular health and fitness after stroke," Topics in Stroke Rehabilitation, vol. 12, no. 1, pp. 1-16, 2005.

[5] U. Svantesson, H. Takahashi, U. Carlsson, A. Danielsson, and K. S. Sunnerhagen, "Muscle and tendon stiffness in patients with upper motor neuron lesion following a stroke," European Journal of Applied Physiology, vol. 82, no. 4, pp. 275-279, 2000.

[6] R. L. Waters and S. Mulroy, "The energy expenditure of normal and pathologic gait," Gait and Posture, vol. 9, no. 3, pp. 207-231, 1999.

[7] M. M. Platts, D. Rafferty, and L. Paul, "Metabolic cost of overground gait in younger stroke patients and healthy controls," Medicine and Science in Sports and Exercise, vol. 38, no. 6, pp. 1041-1046, 2006.

[8] A. Maeda, T. Yuasa, K. Nakamura, S. Higuchi, and Y. Motohashi, "Physical performance tests after stroke: reliability and validity," American Journal of Physical Medicine and Rehabilitation, vol. 79, no. 6, pp. 519-525, 2000.

[9] K. M. Michael, J. K. Allen, and R. F. MacKo, "Reduced ambulatory activity after stroke: the role of balance, gait, and cardiovascular fitness," Archives of Physical Medicine and Rehabilitation, vol. 86, no. 8, pp. 1552-1556, 2005. 
[10] N. F. Gordon, M. Gulanick, F. Costa et al., "Physical activity and exercise recommendations for stroke survivors: an American Heart Association scientific statement from the Council on Clinical Cardiology, Subcommittee on Exercise, Cardiac Rehabilitation, and Prevention; the Council on Cardiovascular Nursing; the Council on Nutrition, Physical Activity, and Metabolism; and the Stroke Council," Stroke, vol. 35, no. 5, pp. 1230-1240, 2004.

[11] J. H. Rimmer, B. Riley, E. Wang, A. Rauworth, and J. Jurkowski, "Physical activity participation among persons with disabilities: barriers and facilitators," American Journal of Preventive Medicine, vol. 26, no. 5, pp. 419-425, 2004.

[12] S. Mudge and N. S. Stott, "Outcome measures to assess walking ability following stroke: a systematic review of the literature," Physiotherapy, vol. 93, no. 3, pp. 189-200, 2007.

[13] D. Rand, J. J. Eng, P. F. Tang, C. Hung, and J. S. Jeng, "Daily physical activity and its contribution to the healthrelated quality of life of ambulatory individuals with chronic stroke," Health and Quality of Life Outcomes, vol. 8, article 80, 2010.

[14] A. Bjorkdahl, A. L. Nilsson, G. Grimby, and K. S. Sunnerhagen, "Does a short period of rehabilitation in the home setting facilitate functioning after stroke? A randomized controlled trial," Clinical Rehabilitation, vol. 20, no. 12, pp. 1038-1049, 2006.

[15] K. S. Sunnerhagen, M. Hedberg, G. B. Henning, A. Cider, and U. Svantesson, "Muscle performance in an urban population sample of 40- to 79-year-old men and women," Scandinavian Journal of Rehabilitation Medicine, vol. 32, no. 4, pp. 159-167, 2000.

[16] R. O. Crapo, R. Casaburi, A. L. Coates et al., "ATS statement: guidelines for the six-minute walk test," American Journal of Respiratory and Critical Care Medicine, vol. 166, supplement 1, pp. 111-117, 2002.

[17] G. Borg, "Psychophysical scaling with applications in physical work and the perception of exertion," Scandinavian Journal of Work, Environment and Health, vol. 16, no. 1, pp. 55-58, 1990.

[18] P. L. Enrichi and D. L. Sherrill, "Reference equations for the six-minute walk in healthy adults," American Journal of Respiratory and Critical Care Medicine, vol. 158, no. 5, pp. 1384-1387, 1998.

[19] J. MacGregor, "The evaluation of patient performance using long-term ambulatory monitoring technique in the domiciliary environment," Physiotherapy, vol. 67, no. 2, pp. 30-33, 1981.

[20] A. Danielsson, C. Willén, and K. S. Sunnerhagen, "Measurement of energy cost by the physiological cost index in walking after stroke," Archives of Physical Medicine and Rehabilitation, vol. 88, no. 10, pp. 1298-1303, 2007.

[21] E. Fredrickson, R. L. Ruff, and J. J. Daly, "Physiological Cost Index as a proxy measure for the oxygen cost of gait in stroke patients," Neurorehabilitation and Neural Repair, vol. 21, no. 5, pp. 429-434, 2007.

[22] O. Sangha, G. Stucki, M. H. Liang, A. H. Fossel, and J. N. Katz, "The Self-Administered Comorbidity Questionnaire: a new method to assess comorbidity for clinical and health services research," Arthritis Care and Research, vol. 49, no. 2, pp. 156163, 2003.

[23] A. R. Fugl-Meyer, L. Jaasko, and I. Leyman, "The post stroke hemiplegic patient. I. A method for evaluation of physical performance," Scandinavian Journal of Rehabilitation Medicine, vol. 7, no. 1, pp. 13-31, 1975.
[24] K. Hagberg, E. Häggström, and R. Brånemark, "Physiological cost index (PCI) and walking performance in individuals with transfemoral prostheses compared to healthy controls," Disability and Rehabilitation, vol. 29, no. 8, pp. 643-649, 2007.

[25] R. A. Washburn, K. W. Smith, A. M. Jette, and C. A. Janney, "The Physical Activity Scale for the Elderly (PASE): development and evaluation," Journal of Clinical Epidemiology, vol. 46, no. 2, pp. 153-162, 1993.

[26] K. A. Martin, W. J. Rejeski, M. E. Miller, M. K. James, W. H. Ettinger, and S. P. Messier, "Validation of the PASE in older adults with knee pain and physical disability," Medicine and Science in Sports and Exercise, vol. 31, no. 5, pp. 627-633, 1999.

[27] L. H. Krarup, T. Truelsen, A. Pedersen et al., "Level of physical activity in the week preceding an ischemic stroke," Cerebrovascular Diseases, vol. 24, no. 2-3, pp. 296-300, 2007.

[28] B. Camarri, P. R. Eastwood, N. M. Cecins, P. J. Thompson, and S. Jenkins, "Six minute walk distance in healthy subjects aged 55-75 years," Respiratory Medicine, vol. 100, no. 4, pp. 658$665,2006$.

[29] U. B. Flansbjer, A. M. Holmbäck, D. Downham, C. Patten, and J. Lexell, "Reliability of gait performance tests in men and women with hemiparesis after stroke," Journal of Rehabilitation Medicine, vol. 37, no. 2, pp. 75-82, 2005.

[30] K. Hagberg, R. Tranberg, R. Zügner, and A. Danielsson, "Reproducibility of the physiological cost index among individuals with a lower-limb amputation and healthy adults," Physiotherapy Research International, vol. 16, no. 2, pp. 92$100,2011$.

[31] L. F. Teixeira-Salmela, R. Devaraj, and S. J. Olney, "Validation of the human activity profile in stroke: a comparison of observed, proxy and self-reported scores," Disability and Rehabilitation, vol. 29, no. 19, pp. 1518-1524, 2007.

[32] A. G. Karatepe, R. Gunaydin, T. Kaya, and G. Turkmen, "Comorbidity in patients after stroke: impact on functional outcome," Journal of Rehabilitation Medicine, vol. 40, no. 10, pp. 831-835, 2008.

[33] I. G. L. van de Port, G. Kwakkel, I. van Wijk, and E. Lindeman, "Susceptibility to deterioration of mobility long-term after stroke: a prospective cohort study," Stroke, vol. 37, no. 1, pp. 167-171, 2006.

[34] M. Wen, N. R. Kandula, and D. S. Lauderdale, "Walking for transportation or leisure: what difference does the neighborhood make?" Journal of General Internal Medicine, vol. 22, no. 12, pp. 1674-1680, 2007. 


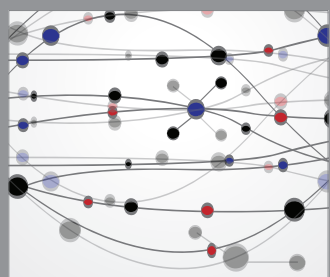

The Scientific World Journal
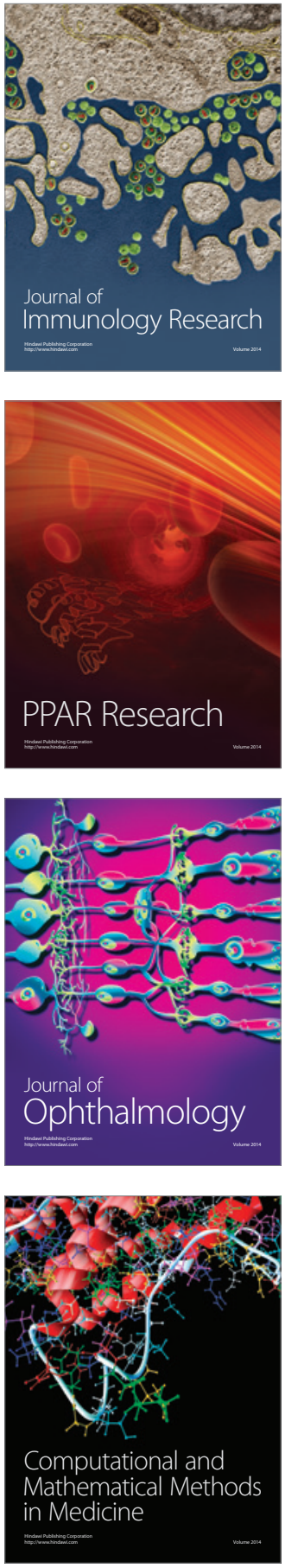

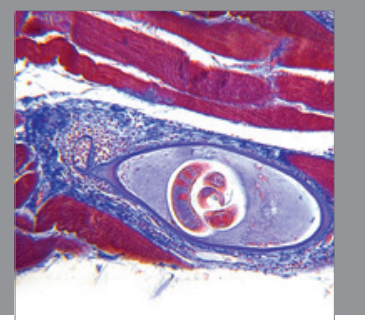

Gastroenterology

Research and Practice
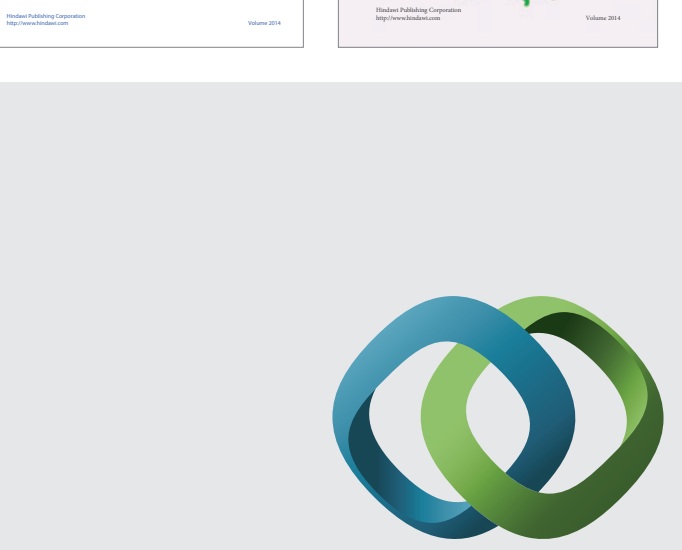

\section{Hindawi}

Submit your manuscripts at

http://www.hindawi.com
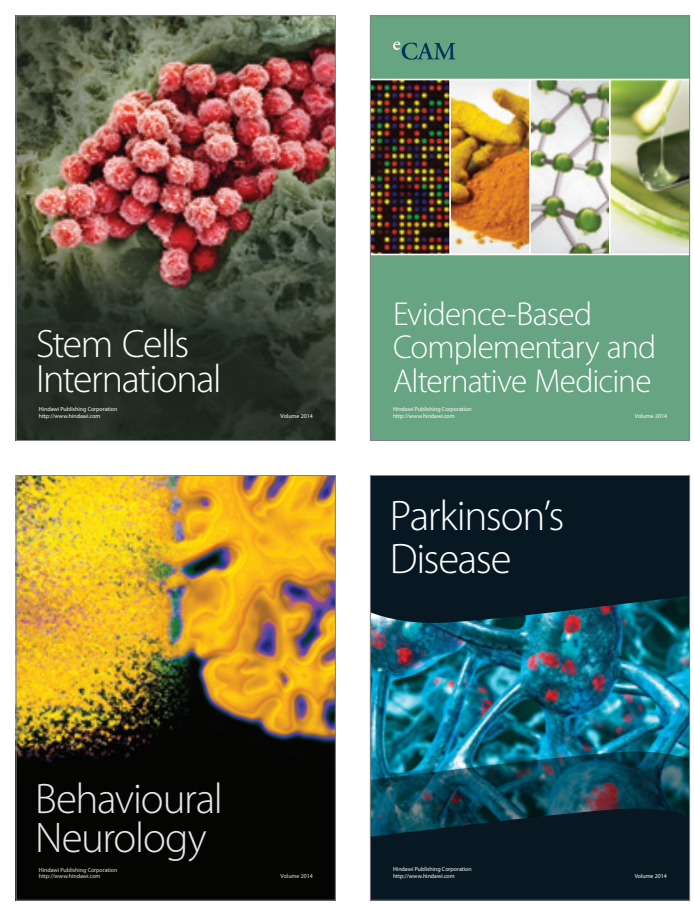

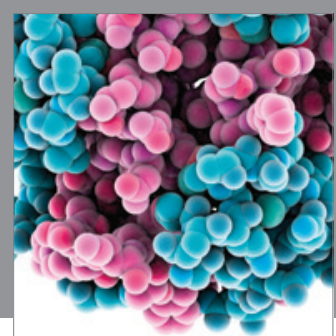

Journal of
Diabetes Research

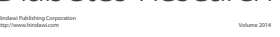

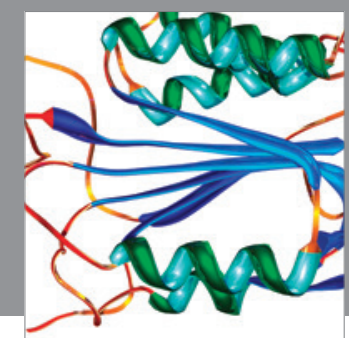

Disease Markers
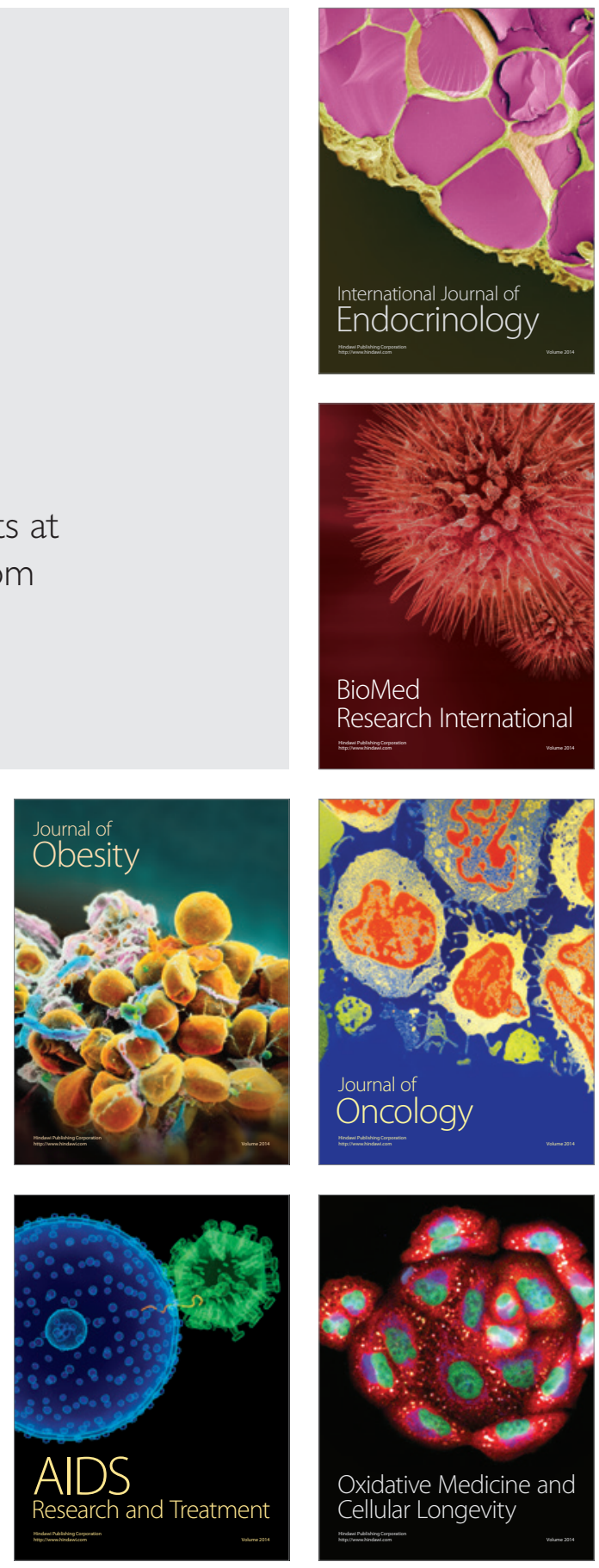